\title{
Probability Distribution of Cross-sectional radius of Corroded Steel Bars in Concrete and Its Application
}

\author{
LI Chongkai ${ }^{1}$, ZHANG Weiping ${ }^{1, *}$, GU Xianglin ${ }^{1}$, and HUANG Qinghua ${ }^{1}$
}

${ }^{1}$ Key Laboratory of Performance Evolution and Control for Engineering Structures (Tongji University), Ministry of Education, Shanghai, 200092, China

\begin{abstract}
The impress current method is applied to acquire corroded steel bars embedded in concrete, and three-dimensional(3D) laser scanning techniques are applied on corroded steel bars to obtain the cross-sectional radius of corroded steel bars. Statistical analysis shows that with the increase of corrosion degree, the variation of radius of corroded steel bars increases linearly. For different types of steel bars, plain round steel bars have a larger sensitivity to corrosion than ribbed ones. Original radius of steel bars and mixture proportion of concrete have negligible effect on the variability of radius of corroded steel bars. A normal distribution model is obtained to describe radius data of corroded steel bars. For the convenience of practical application, the indicator, $R$, which is the ratio of the average to the minimum cross-sectional areas of corroded steel bars, is introduced to quantify the longitudinal variation of the cross-sectional areas. By using Monte-Carlo simulation, the indicator, $\mathrm{R}$, of corroded steel bars are achieved based on the probability distribution of radius. The indicator $\mathrm{R}$ can be fitted well by the Gumbel distribution, and the distribution parameters increase linearly with the increases of corrosion degree.
\end{abstract}

\section{Introduction}

Corrosion of steel bars in concrete has become one of the predominant factors leading to performance degradation of concrete structures. Corrosion-related durability accidents have resulted in great economic losses [1-3]. Due to the heterogeneity of the surrounding environment and concrete materials, as well as the spatial variation of the thickness of concrete cover, the corrosion process of the steel bars is essentially a stochastic process. Once corrosion initiates on steel bars, the cross-sectional areas of a corroded steel bar vary along its longitudinal axis. Thus, a corroded reinforced concrete beam may fail at an uncritical section with a smaller cross-sectional area which is not subjected to the maximum moment, and the failure probability of the beam may increase. In order to investigate the impact of the longitudinal variation of cross-sectional areas on mechanical properties of steel bars and to predict failure probability of reinforced concrete members, variability of cross-sectional areas of corroded steel bars should be investigated.

The variation of the cross-sectional area of a corroded rebar along its length can be quantified by either one of the following two indicators, i.e. the pitting factor $R_{p}=p_{\max } / p_{\text {av }}$ or the spatial heterogeneity factor of cross-sectional area $R=A_{\text {av }} / A_{\text {min }}$, where $p_{\max }$ is the maximum pit depth of corrosion pits; $p_{\text {av }}$ is the average pit depth of corrosion pits, and, $A_{\text {av }}$ and $A_{\min }$ are the average and minimum cross-sectional area of corroded rebars, respectively. For corroded rebars of various diameters, lengths and corrosion degrees, the average value of $R_{\mathrm{p}}$ ranged from 4.0 to 23.8 and its variability can be characterized by the Gumbel distribution (e.g., [7-7]). To calculate the load bearing capacity and failure probability of corroded RC members, the calculation of the minimum cross-sectional area is of vital importance. To meet the convenience of engineers, the indicator $R$ was adopted to determine directly the minimum crosssectional area, regardless of complicated corrosion topography of the rebar $[9,10]$. Statistical analysis found that the indicator $R$ of corroded rebar can be characterized by Gumbel distribution and both the location parameter and the scale parameter increased linearly with increase in the corrosion degree [10].

The cross-sectional radius of corroded steel bars are investigated in this paper instead of getting crosssectional areas because of the small sample size of areas. By analyzing influencing coefficients, the stochastic process model of cross-sectional radius of corroded steel bars is established. Cross-sectional areas and indicator, $R$, are obtained by methematical calculation with the data of radius which is generated through Monte-Carlo simulation according to the stochastic process. Then the probability distribution and influence factors of indicator, $R$, are analyzed.

\section{Preparation of corroded steel bars}

Steel bars, with the nominal diameter of $10 \mathrm{~mm}, 14 \mathrm{~mm}$, $20 \mathrm{~mm}$ and the strength grade of HPB235, HRB335,

* Corresponding author: weiping_zh@tongji.edu.cn 
HRB400, were cast into concrete slabs with the size of $600 \times 350 \times 100 \mathrm{~mm}$ and two different mixture proportions. Each slab was reinforced with six steel bars, and the thickness of concrete cover was $25 \mathrm{~mm}$. Both ends of each steel bar were extended $50 \mathrm{~mm}$ beyond the slabs for connection with electric wires. After 28 days of curing, the specimens were subjected to accelerated corrosion by applying anodic current, which is proved a efficient method to obtain corroded steel rebar specimens[7,10]. Fig. 1 shows the in-situ setup of accelerated corrosion. Specimens were divided into 6 groups with different taget average weight loss ratios, ranging from 0.05 to 0.30. The corresponding duration of the applied corrosion current was estimated by Faraday's law[11].

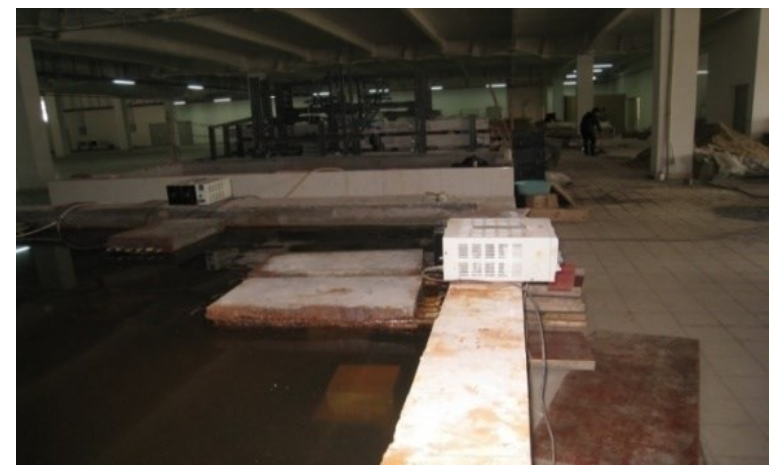

Fig. 1. In-situ setup of accelerated corrosion.

After the completion of each corrosion test, the slabs were broken and the corroded steel bars were retrieved and collected. All of the corroded specimens underwent acid cleaning and drying according to ASTM G1-03 [12]. Fig. 2 shows corroded steel bars after acid cleaning and drying. Then average weight loss ratio of each corroded steel bars was recorded.

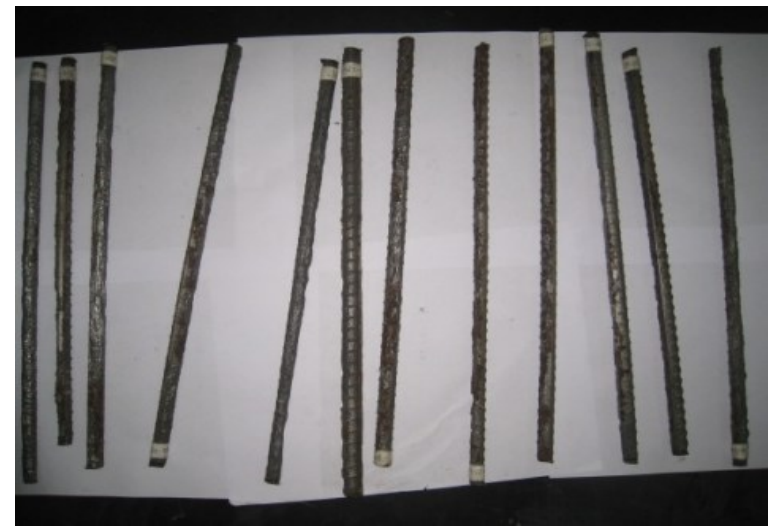

Fig. 2. Part of corroded steel bars after acid cleaning and drying

\section{Cross-sectional radius of corroded steel bars}

Geometric models of all corroded steel bars after acid cleaning and drying were built using a 3D laser scanner, as shown in Fig. 3. By using Geomagic Studio software ${ }^{\circledR}$ and Unigraphics NX3.0 software ${ }^{\circledR}$, 3D coordinates of each point on the surface of the corroded steel bars can be obtained, and the $3 \mathrm{D}$ geometric model can be finally constructed with satisfactory accuracy [10].

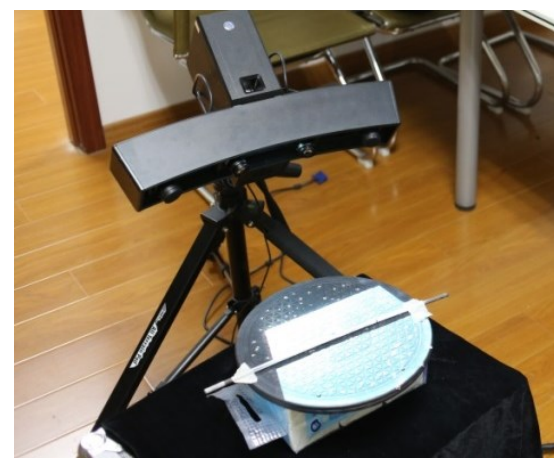

Fig. 3. Three-dimensional laser scanner.

Cross-sectional radius, r, were obtained by calculating coordinates of each point on the geometric model of a corroded steel bar with an element length of $100 \mathrm{~mm}$. By establishing the central axis of uncorroded part of each corroded steel bar, the axis of corroded steel bars was determined, as shown in Fig. 4. The axis of the geometric model of a steel bar was moved to x-axis, so the centre of each cross section was the original point in $y-z$ plane. The corresponding cross-sectional radius can be calculated by Eq.(1).

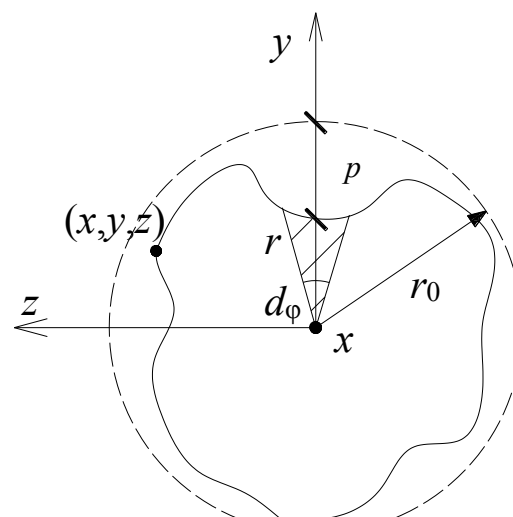

Fig. 4. Schematic calculation of cross-sectional area.

$$
r=\sqrt{y^{2}+z^{2}}
$$

Suppose that a steel bar is divided into $n$ elements and the element length, $l$, is so small that one element can be represented by that with the same cross-sectional profile. The original nominal radus of a steel bar is supposed to be a constant. Then the average weight loss ratio of a corroded steel bar can be calculated by Eq.(2). It is obvious that the relationship among the mean value, the variation of radius and the average corrosion degree of corroded steel bars of a certain length can be described with an identical equation. The measured data can also prove that result, as show in Fig. 5.

$\eta_{s}=1-\frac{\sum_{1}^{n} \rho A_{i} l}{\rho A_{0} L}=1-\frac{\sum_{1}^{n} \sum_{1}^{m} r_{i j}^{2}}{n m r_{0}^{2}}=1-\frac{E\left(r_{k}^{2}\right)}{r_{0}^{2}}=1-\frac{\mu^{2}+\sigma^{2}}{r_{0}^{2}}$ 
where $\rho$ is the density of steel; $A_{0}, r_{0}$ are the original nominal cross-sectional area and radius of steel barrespectively; $A, r$ are cross-sectional area and redius of corroded steel bar, and the subscript represents the element number; $m$ is the number of $r$ obtained from one cross section; $\mu, \sigma$ are the mean value and standard deviation of $r$ respectively.

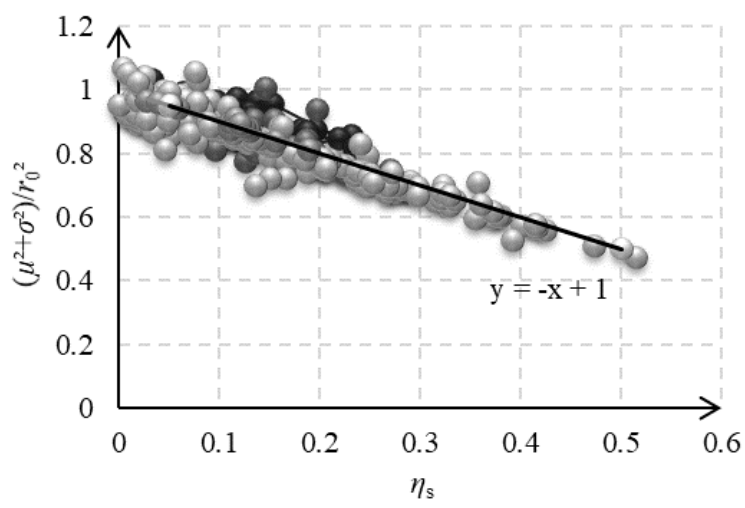

Fig. 5. Relationship between $\left(\mu^{2}+\sigma^{2}\right) / r_{0}^{2}$ and $\eta_{\mathrm{s}}$ obtained from measured data of $r$.

\subsection{Probability distribution of radius}

It was found that the variability of pit depth, $p$, on pipelines or steel plates can be characterized by normal distribution (e.g., [13-15]). Similarly the cross-sectional radius of corroded steel bars can also be characterized by normal distribution according to Eq.(3), which can also be verified by statistical analysis of measured crosssectional radius. For steel bars with a given corrosion degree, the probability distribution density can be quantified by Eq.(4).

$$
\begin{gathered}
r=r_{0}-p \\
f(r)=\frac{1}{\sigma \sqrt{2 \pi}} \exp \left\{-\frac{(r-\mu)^{2}}{2 \sigma^{2}}\right\}
\end{gathered}
$$

\subsection{Probability distribution parameters analysis}

\subsubsection{Influence of corrosion degree}

With the increase of corrosion degree, the variance of cross-sectional radius of corroded steel bars increases linearly, as show in Fig. 6. The relationship between the variance and the average weight loss ratio can be described by Eq.(5).

$$
\sigma^{2}=k \eta_{s}+a_{k}
$$

where $a_{\mathrm{k}}$ is the variance of uncorroded steel bars.

According to Eq.(2), the mean value of crosssectional radius of corroded steel bars can be calculated by Eq.(6).

$$
\mu^{2}=r_{0}^{2}-a_{k}-\left(r_{0}^{2}+k\right) \eta_{s}
$$

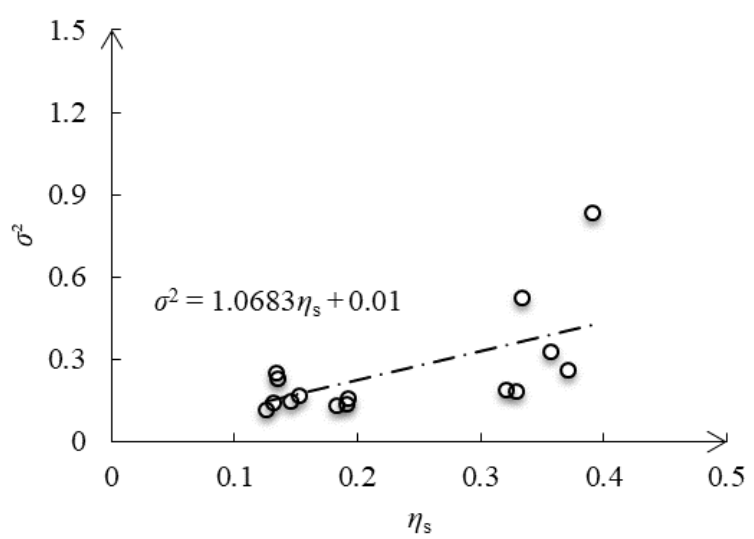

Fig. 6. Relationship between $\sigma^{2}$ and $\eta_{\mathrm{s}}$ of corroded steel bars (steel type: HRB335, original nominal diameter: $14 \mathrm{~mm}$, and all the mixture proportions of slabs are the same).

\subsubsection{Influence of original nominal diameter}

Fig. 7 shows the variance of cross-sectional radius of corroded steel bars with different original nominal diameters, while other experiment condition is the same. For corroded steel bars obtained by the impressed current method, the original nominal diameter has negligible effect on the variability of cross-sectional radius.

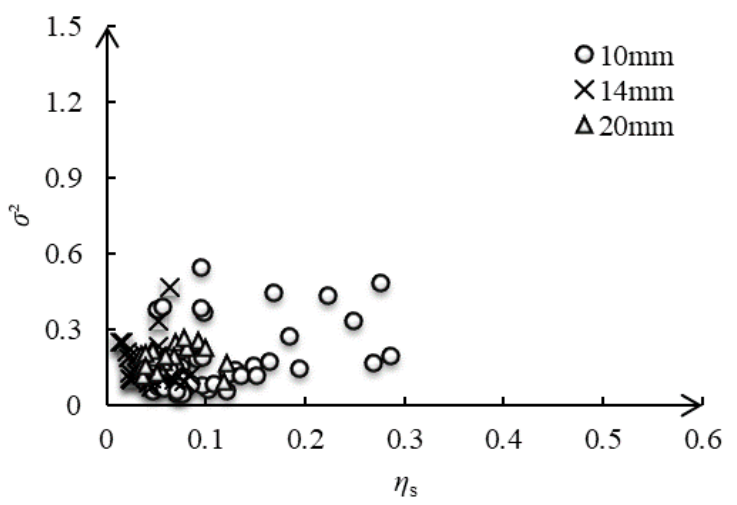

Fig. 7. $\sigma^{2}$ of corroded steel bars with different original nominal diameters.

\subsubsection{Influence of steel bar type}

Different types of steel bars with different surface morphology, plain round or ribbed, will have different sensitivity to corrosion. As Fig. 8 shows, plain round steel bars have a larger sensitivity to corrosion than ribbed steel bars.

\subsubsection{Influence of mixture proportion of concrete}

Statistical analysis of cross-sectional radius of corroded steel bars, which are obtained from concrete slabs with different mixture proportions, shows that mixture proportions of concrete has little influence on the variability of cross-sectional radius, as show in Fig. 9. 


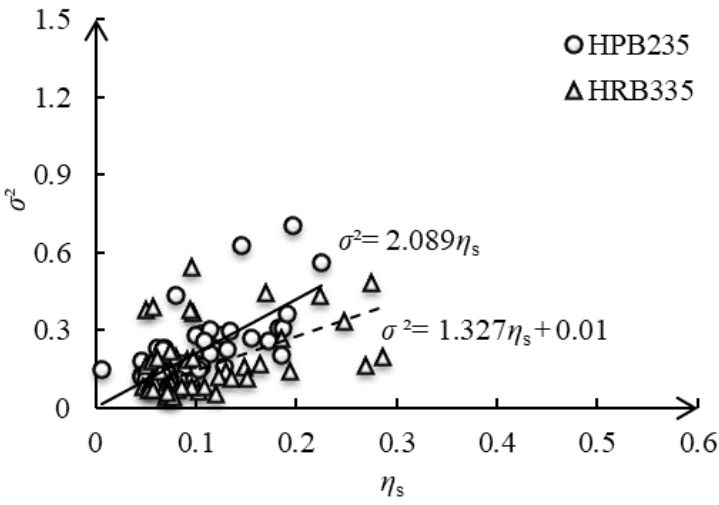

Fig. 8. $\sigma^{2}$ of corroded steel bars of different surface morphology.

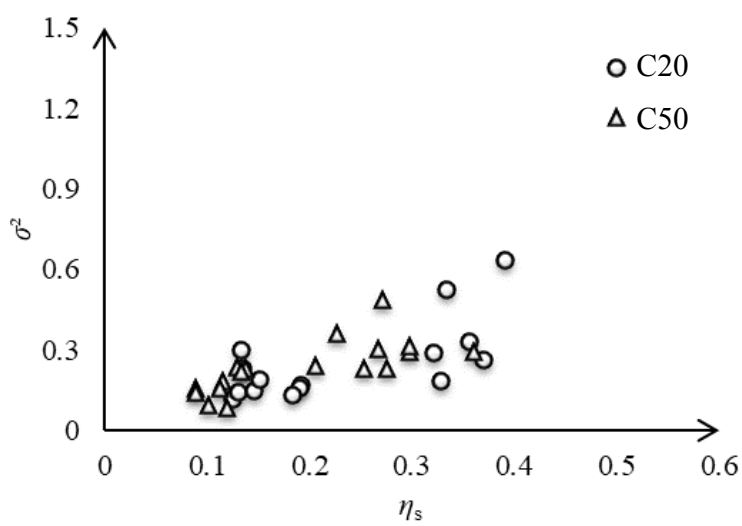

Fig. 9. $\sigma^{2}$ of corroded steel bars obtained from concrete slabs with different mixture proportions.

\subsection{Stochastic process model}

Consider all the discussion in section 3.1 and 3.2, the variance of cross-sectional radius of corroded steel bars can be calculated by a multiparameter formula, Eq.(7).

$$
\sigma^{2}=\mathrm{C}\left(a+b i_{c o r r}\right) \eta_{s}+a_{k}
$$

where $C$ is the coefficient of steel bar type; $i_{\text {corr }}\left(\mathrm{mA} / \mathrm{cm}^{2}\right)$ is the current density; $a, b$ are fitting parameters.

$a_{\mathrm{k}}$ is the variance of uncorroded steel bars, which is acquired by statistical analysing the rib length and width and the interval length between adjacent ribs. The value of $a_{\mathrm{k}}$ is 0 for plain round bar, 0.01 for HRB335 steel bar, 0.1 for HRB400 steel bar.

Surface fitting Eq.(7) is conducted by using Matlab ${ }^{\circledR}$ with cross-sectional radius data of corroded steel bars. The fitting results are shown in Table 1.

The mean value, $\mu$, can be calculated by Eq.(6), where $k=C\left(a+b i_{\text {corr }}\right)$. The stochastic process model of cross-sectional radius can be characterized by Eq.(8).

$$
f\left(r\left(\eta_{s}\right)\right)=\frac{1}{\sqrt{2 \pi} \sigma\left(\eta_{s}\right)} \exp \left(-\frac{\left[r\left(\eta_{s}\right)-\mu\left(\eta_{s}\right)\right]^{2}}{2 \sigma^{2}\left(\eta_{s}\right)}\right)
$$

Table 1. Coefficients of Eq.(7)

\begin{tabular}{|c|c|c|}
\hline Coefficient & Value & Range of application \\
\hline \multirow{2}{*}{$C$} & 1.283 & HPB235 \\
\cline { 2 - 3 } & 1 & HRB335 \\
\cline { 2 - 3 } & 0.954 & HRB400 \\
\hline \multirow{2}{*}{$a$} & 0.8075 & $/$ \\
\hline \multirow{2}{*}{$b$} & 4.216 & $/$ \\
\hline \multirow{2}{*}{$a_{\mathrm{k}}$} & 0.01 & Plain round bar \\
\cline { 2 - 3 } & 0.1 & HRB335 \\
\cline { 2 - 3 } & & HRB400 \\
\hline
\end{tabular}

\section{Variation of cross-sectional areas}

For corroded steel bars, using commercial software, ProEngineer ${ }^{\circledR}$, the areas of the discrete cross sections were obtained along the longitudinal axis at intervals of $2 \mathrm{~mm}$, as shown in Fig. 10.

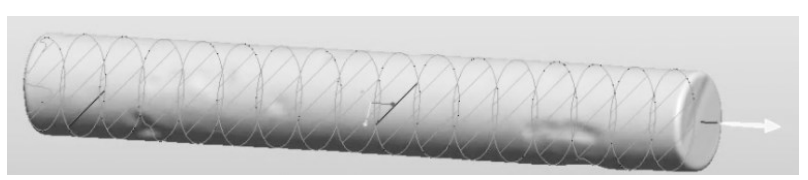

Fig. 10. Calculation diagram of the cross-sectional area of a corroded steel bar

To enlarge the sample size of the cross-sectional area, $A$, of corroded steel bars, Monte-Carlo simulation was carried on by using Matlab ${ }^{\circledR}$, based on the stochastic process model of cross-sectional radius(Eq.(8)), to generate radius data. Then cross-sectional areas were calculated by summating the areas of $n$ sectors with equal radian, $d_{\varphi}=2 \pi / n$, as shown in Fig. 4 and Eq.(9), where $n=72$.

$$
A=\sum_{j=1}^{n} \frac{1}{2} d_{\varphi} r_{j}^{2}
$$

For each average weight loss ratio, $m$ cross-sectional areas were generated corresponding to the element length of $2 \mathrm{~m} \mathrm{~mm}$ and 1000 elements were generated to obtain the indicator $R$. Statistical analysis of the indicator $R$ was conducted. Fig. 11 shows typical probability histograms of $R$ with an element length of $50 \mathrm{~mm}$.

\subsection{Probability distribution of $R$}

To verify whether $R$ can be fitted to a Gumbel distribution, the values of $R$ for each average weight loss ratio were accumulated and sorted from small to large, and the Kolmogorov-Smirnov (K-S) test was conducted $[10,16]$. For the $i^{\text {th }}$ value of $R$, as designated by $R_{i}$, the 
corresponding empirical probability of occurrence $F_{i}$ can be calculated as Eq.(10).

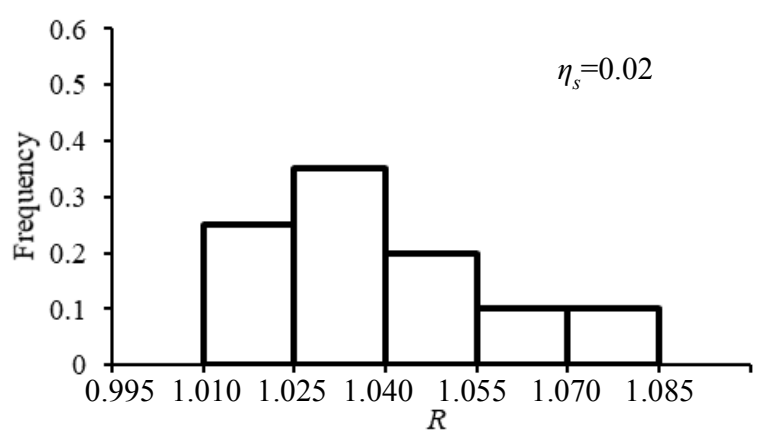

Fig. 11. Typical probability histograms of $R$

$$
F_{i}=i /(N+1) \quad i=1,2, \ldots N
$$

Fig.12 shows the test results of $R$. The probability density function can be expressed as Eq.(11).

$$
f(R)=\mathrm{e}^{-\left(R-\mu_{R}\right) / \sigma_{R}} \exp \left[-\mathrm{e}^{-\left(R-\mu_{R}\right) / \sigma_{R}}\right] / \sigma_{R}
$$

where $\mu_{\mathrm{R}}$ is the location parameter of the Gumbel distribution; and $\sigma_{\mathrm{R}}$ is the scale parameter of the Gumbel distribution.

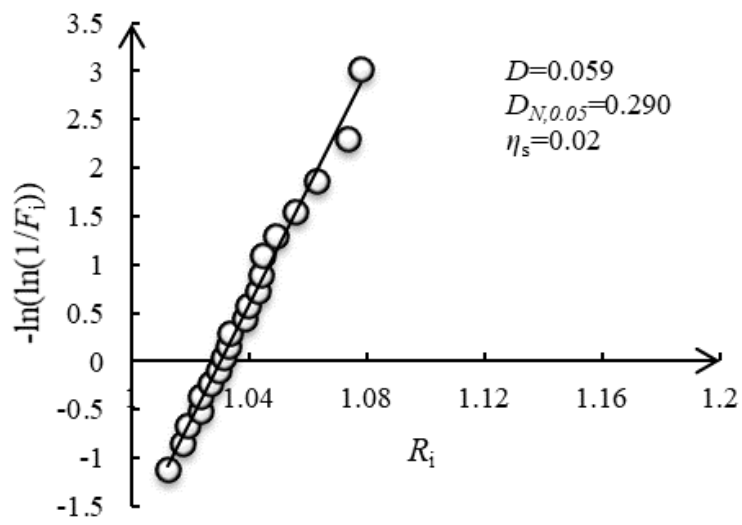

Fig. 12. Relationship between values of $-\ln \left(\ln \left(1 / F_{\mathrm{i}}\right)\right)$ and $R_{\mathrm{i}}$

According to the expectation and variance of $R$, namely $\mathrm{E}(R)$ and $\mathrm{D}(R), \mu_{\mathrm{R}}, \sigma_{\mathrm{R}}$ can be evaluated as Eq.(12):

$$
\left\{\begin{array}{l}
E(R)=\mu_{R}+\gamma \sigma_{R} \\
D(R)=\pi^{2} \sigma_{R}^{2} / 6
\end{array}\right.
$$

where $\gamma=$ a Euler's constant of 0.5772 [16].

Fig. 13 shows the relationship between the Gumbel statistical parameters of $R$ and the average weight loss ratio, $\eta_{s}$. With an increase in the average weight loss ratio, the distribution parameters, location parameter and scale parameter, increase linearly and the results of experimental measure and Monte-Carlo simulation match well.
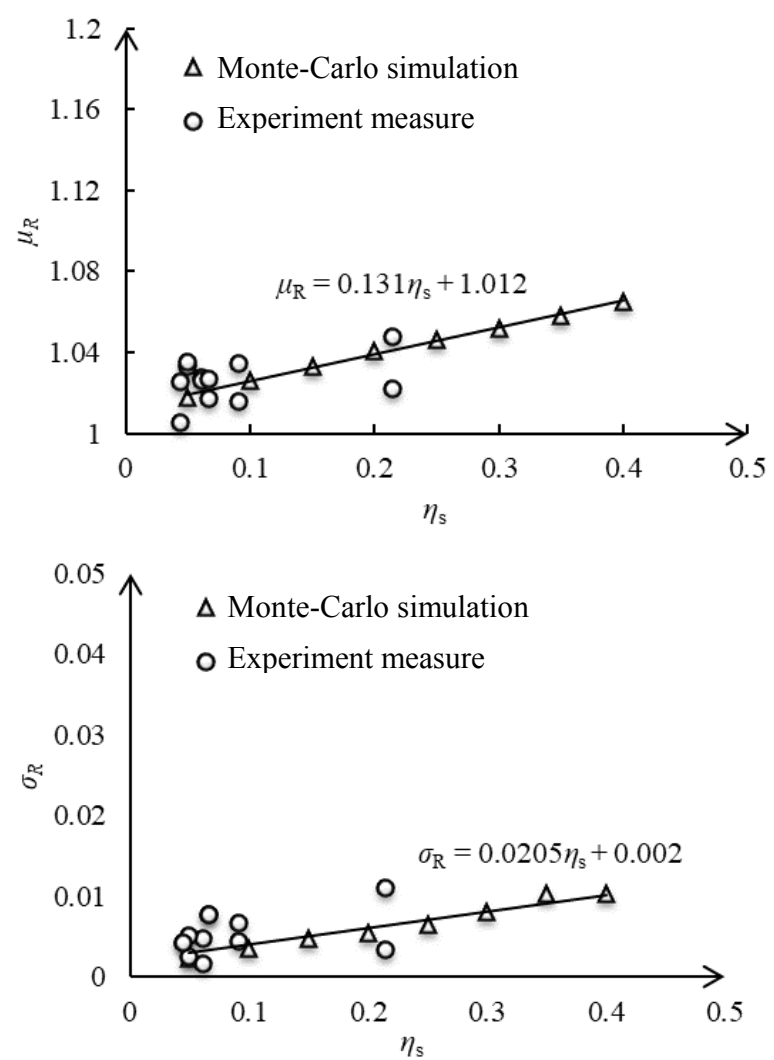

Fig. 13 Relationship between the Gumbel statistical parameters of $R$ and $\eta_{s}$ (steel bar type: HRB335, original nominal diameter:20mm, element length: $100 \mathrm{~mm}$ )

\subsection{Influencing factors}

\subsubsection{Influence of element length}

The influence of element length on probability distribution of $R$ was analyzed by Monte-Carlo simulation. For each certain element length, MonteCarlo simulation data of $A$ was random divided into 100 groups, and the data size of each group represented the certain element length (e.g. for the element length of $50 \mathrm{~mm}$, the data size of each group was 50). The theoretical relationship between Gumbel statistical parameters of $R$ and element length is characterized by Eq.(13).

$$
\left\{\begin{array}{l}
\mu_{R}=\mu_{0}+\sigma_{0} \ln \left(l / l_{0}\right) \\
\sigma_{R}=\sigma_{0}
\end{array}\right.
$$

where $l_{0}$ is the element length for a benchmark probability distribution of $R, \mu_{0}, \sigma_{0}$ are the distribution parameters corresponding to $l_{0}, l$ is the element length.

The results of the relationship between Gumbel statistical parameters of $R$ and element length obtained from Monte-Carlo simulation and theoretical derivation match well, as show in Fig. 14. So The relationship between $\mu_{R}$ and $l$ can be characterized by a logarithmic linear correlation and $\sigma_{R}$ is independent with $l$. 

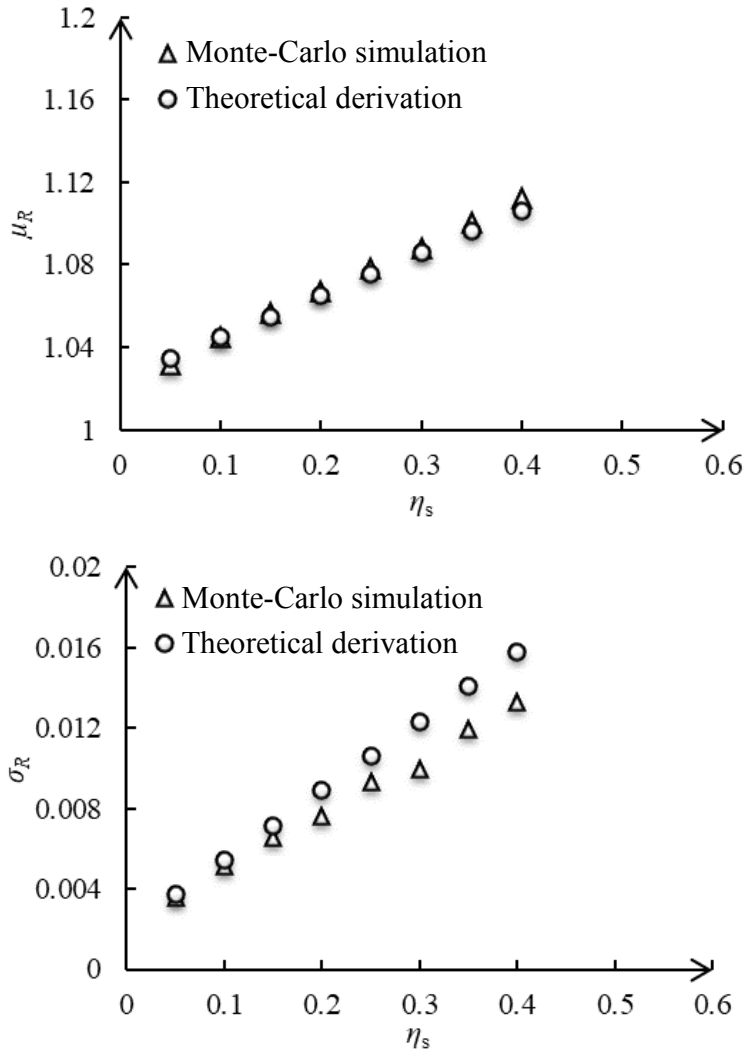

Fig. 14 Relationship between the Gumbel statistical parameters of $R$ and $\eta_{s}$ (element length: $300 \mathrm{~mm}$ )

\subsubsection{Influence of original nominal diameter}

As Fig. 15 shows, for corroded steel bars with the same applied current density and type, the increase of original nominal diameter will lead to the decrease of distribution parameters of indicator $R$. That means corroded steel bars with larger original nominal diameter will have a smaller variability of cross-sectional areas.

\subsubsection{Influence of steel bar type}

For different types of steel bars, the relationship between Gumbel statistical parameters of $R$ and $\eta_{s}$ is presented in table 2. As the surface morphology of steel bars changing from plain round to ribbed, the slope of the regressive funtion decreases significantly. That means plain round steel bars are more sensitive to corrosion. As the strength grade increases, the slope of the regressive function decrease a little. That can conclude that high strength steel may have advantages to resist corrosion.

Table 2. Regressive functions of Gumbel statistical parameters

\begin{tabular}{|c|c|c|}
\hline Type & $\boldsymbol{\mu}_{\boldsymbol{R}}$ & $\boldsymbol{\sigma}_{\boldsymbol{R}}$ \\
\hline $\mathrm{HPB} 235$ & $\mu_{R}=0.292 \eta_{\mathrm{s}}+1$ & $\sigma_{R}=0.044 \eta_{\mathrm{s}}$ \\
\hline $\mathrm{HRB} 335$ & $\mu_{R}=0.181 \eta_{\mathrm{s}}+1$ & $\sigma_{R}=0.031 \eta_{\mathrm{s}}+0.002$ \\
\hline HRB400 & $\mu_{R}=0.162 \eta_{\mathrm{s}}+1$ & $\sigma_{R}=0.026 \eta_{\mathrm{s}}+0.004$ \\
\hline
\end{tabular}
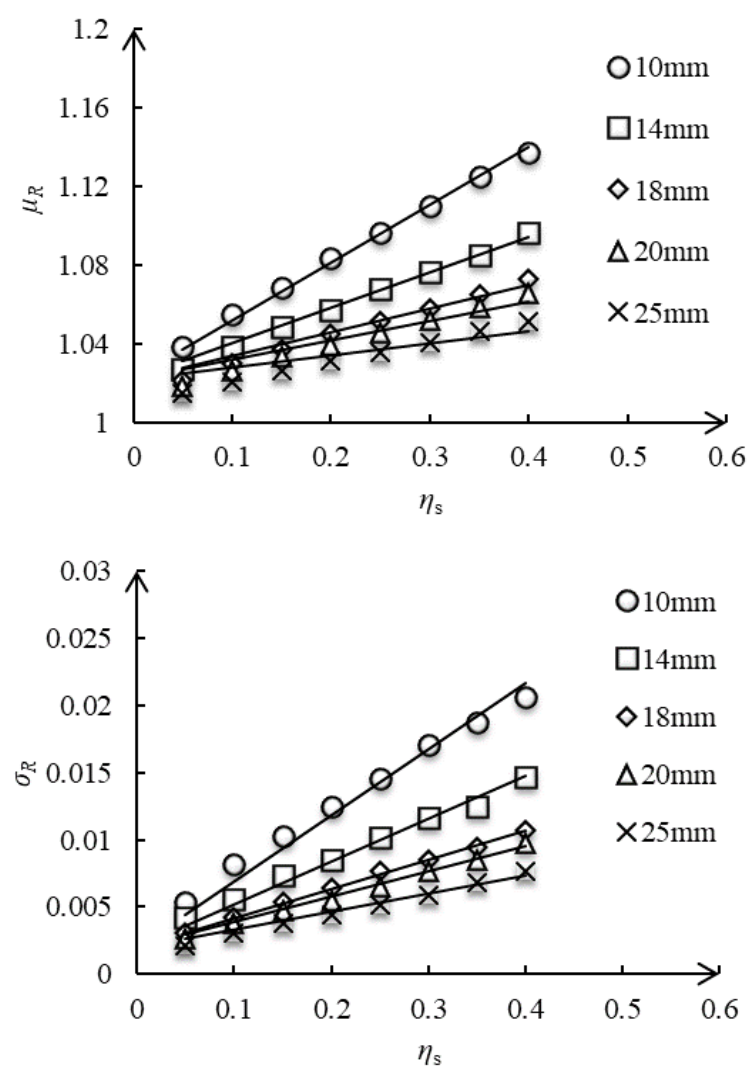

Fig. 15 Relationship between the Gumbel statistical parameters of $R$ and $\eta_{s}$ (steel type: HRB335)

\section{Conclusions}

For cross-sectional radius of corroded steel bars, a unified equation is derived to describe the relationship of the mean value, the variation of radius and the average corrosion degree of corroded steel bars of a certain length. With the increase of corrosion degree, the variation of radius of corroded steel bars increases linearly. Plain round steel bars will have a larger sensitivity to corrosion than ribbed ones. Original radius of steel bars and mixture proportion of concrete have negligible effect on the variability of radius of corroded steel bars. Then the stochastic process model of radius was established.

For the indicator $R$, it can be fitted by Gumbel distribution and the distribution parameters increase linearly with the increases of corrosion degree. With increase in the element length, the location parameter increases significantly, while the scale parameter is independent on the element length. The relationship between the location parameter and the element length can be characterized by a logarithmic linear correlation.

Acknowledgements: This research project was financially supported by the National Natural Science Foundation of China (Grant No. 51320105013 and 51578402).

\section{References}

1. Li, C. Q., R. E. Melchers, ACI Struct.J., 102(5), 754-762 (2005) 
2. F. Buchhardt, G. Magiera, W. Matthees, et al, CI, 63-68(1984)

3. Y. Mori, B. R. Ellingwood, J. Struct. Eng., 120(8), 824-45(1994)

4. J. A. Gonzalez, C. Andrade, C. Alonso, et al, Cem. Concr. Res., 25(2), 257-264(1995)

5. A. A. Torres-Acosta, M. Martı'Nez-Madrid, J. Mater. Civ. Eng., 15(4), 344-353(2003)

6. M. G. Stewart, Struct. Saf., 26(4), 453-470(2004)

7. M. G. Stewart, A. Al-Harthy, Reliab. Eng. Syst. Saf., 93(3), 373-382(2008)

8. M.S. Darmawan, M. G. Stewart, Struct. Saf., 29(1), 16-31(2007)

9. Wang, X. G., Zhang, W. P., Gu, X. L., et al, Constr. Build. Mater., 38, 846-853(2013)

10. Zhang, W. P., Zhou, B. B., Gu, X. L., et al, J. Mater. Civ. Eng., 26(5), 822-832(2014)

11. T. A. E. Maaddawy, K. A. Soudki, J. Mater. Civ. Eng., 15(1), 41-47 (2003)

12. ASTM G1-03, West Conshohocken, PA.,(2003)

13. D. E. Hawn, Mater. Perform., 16(3), 29-32(1977)

14. M. Ahammed, R. E.Melchers, Eng. Struct., 17(2), 74-80(1995)

15. A. K. Sheikh, J. K. Boah, D. A. Hansen, Corrosion 46(3), 190-197(1990)

16. J. Choi, Appl. Math. Comput. 187(1), 122140(2007) 